\title{
CORRELATION BETWEEN DRYING DEFECTS, THEIR PARAMETERS AND MOISTURE GRADIENT IN KILN-DRIED, SOUTH AFRICAN GROWN EUCALYPTUS GRANDIS POLES*
}

\author{
P. Mugabi ${ }^{1}$, T. Rypstra ${ }^{1}$, H.F. Vermaas ${ }^{1}$, D.G. Nel ${ }^{2}$
}

\begin{abstract}
Non-destructive testing for drying defects in Eucalyptus grandis utility poles would be highly advantageous. These defects can negatively affect creosote preservative treatment and in-service performance. The objective of this study was to assess correlations between drying defects such as surface checking, honeycombing, collapse, their parameters and moisture content (MC) gradient in poles, to possibly find a simple and quick but reliable method to assess internal and external drying defects. Defects and moisture content gradients were measured in 39 kiln-dried E. grandis poles. After measuring surface check length, width and depth using a measuring tape, a ruler and a depth gauge, destructive sampling at the theoretical ground line (TGL) was done to measure the MC gradient between the shell and core of poles. Digital image analysis of cross-sections of discs cut at TGL was used to measure honeycomb check width, length and area, as well as counting individual closed surface checks. Collapse was assessed using qualitative methods. Results showed that honeycombing and collapse were positively, and surface checking and MC gradient were negatively correlated. Surface check width, length and depth were also correlated. Honeycomb count, check width, length and area were strongly correlated. It was concluded that measuring any of these surface check and/ or honeycomb parameters may give meaningful deductions about the extent of surface checking and honeycombing respectively.
\end{abstract}

Keywords: Drying defects, moisture content gradient, Eucalyptus grandis, kiln-drying, South Africa

\section{INTRODUCTION}

Surface checking, honeycombing and collapse are common drying defects in Eucalypt poles. Eucalyptus grandis is highly prone to collapse (Brown 1989), and kiln drying may increase chances of other defects to occur. Chudnoff (1955) noted that during the drying of heavy Eucalyptus poles, the stresses developed in the surface layers are far higher than in thinner members, and collapse is accompanied by severe surface checking and end splitting. Surface checking (Panshin et al. 1964), honeycombing (Wangaard 1950, Garratt 1931) and collapse (Bariska 1992) all affect strength properties of wood.

After drying, it is, therefore, essential that utility poles are inspected for these defects before creosote preservative impregnation. Assessment of all types of drying defects is important, to be certain that acceptable limits are not exceeded. These three different defects or their parameters such as number, dimensions, distribution, position, etc. require different measurement methods. Some parameters are more difficult to assess than others. The South African National Standards specify maximum permissible number, width and length of end and surface checks but not the depth (SANS 754, 2007). Yet in fact, the depth of a surface check may be decisive as far as the success of preservation is concerned, especially if it goes deeper than the sapwood. Unfavourable moisture content (MC) gradient, closed surface checks and honeycombing are also not covered in the specification nor are they comprehensively assessed during pole inspection prior to treatment.

\footnotetext{
*This paper was originally presented at the 2010 EC-IAWS/ESTB7 meeting, Rabat-Morocco, March 2010, and has been updated.

${ }^{1}$ Department of Forest and Wood Science, Private Bag X1, Matieland 7602, Stellenbosch University, South Africa.

${ }^{2}$ Centre for Statistical Consultation, Private Bag X1, Matieland 7602, Stellenbosch University, South Africa.

Corresponding author: tr@sun.ac.za

Received: 28.04. 2010 Accepted: 02.10. 2010.
} 
By determining meaningful correlations between easily quantifiable, non-destructively assessed parameters of externally visible drying defects such as surface check length, width and depth, and parameters quantifying internal defects such as number of closed surface checks, number and area of honeycombs, collapse as well as moisture content gradient, reliable deductions (and predictions) about drying quality may be made without destroying the pole. The specific objectives of the study were to evaluate relationships between:

(i) MC gradient, collapse, surface and honeycomb check parameters;

(ii) surface check length, width and depth, and

(iii) honeycomb check length, width and area.

\section{MATERIALS AND METHODS}

Given the large variation in pole material sources, harvesting, processing variables such as using different drying schedules, kiln design and operational differences in practice, etc., a large sample of poles is required to generate reliable correlations between drying defects, their parameters and moisture content gradient. As a relatively small number of poles were available, the present work was, therefore, more of an exploratory nature.

\section{Timber}

A total of thirty nine debarked E. grandis poles was investigated. Nineteen poles from KwaZulu Natal (KZN) were wrapped in waterproof plastic material and stored at $3^{\circ} \mathrm{C}$ to minimise moisture loss and possible decay. The duration of storage was about one year and 5 months but the poles were still wet and did not show any signs of decay. It was, therefore, assumed that such storage would not affect correlations of drying defects and moisture gradient. The remaining twenty poles were freshly collected from Coetzenburg, Stellenbosch. For all the poles, the diameter at the theoretical ground line (TGL), $1.5 \mathrm{~m}$ from the butt end, ranged between $175-210 \mathrm{~mm}$. Due to the length limitation of the research kiln, only poles $2.1 \mathrm{~m}$ in length could be used. Full length utility pole drying was simulated by sealing and applying end plates to the top-ends. To minimise end splitting, plates were also fixed to freshly cut butt-ends of poles, as is usually done in industry.

\section{Drying}

Pole drying was done in a Kiefer kiln using the following three schedules:

(i) Schedule $1: 80^{\circ} \mathrm{C}$ dry bulb $\left(\mathrm{T}_{\mathrm{db}}\right)$ and $59^{\circ} \mathrm{C}$ wet bulb temperature $\left(\mathrm{T}_{\mathrm{wb}}\right)$ for 8 days. This schedule was used to dry ten poles from KZN and was repeated on ten poles from Coetzenburg, to investigate effect of the source of material;

(ii) Schedule 2: $80^{\circ} \mathrm{C} \mathrm{T}_{\mathrm{db}}$ and $69^{\circ} \mathrm{C} \mathrm{T}_{\mathrm{wb}}$ for 10 days; nine poles from $\mathrm{KZN}$ and

(iii) Schedule 3: $70^{\circ} \mathrm{C} \mathrm{T}_{\mathrm{db}}$ and $59^{\circ} \mathrm{C} \mathrm{T}_{\mathrm{wb}}$ for 12 days; ten poles from Coetzenburg.

Schedule 1 was at the time of this investigation commonly used in the South African wood preservation industry; both schedules 2 and 3 were chosen to create possible milder drying conditions.

The air velocity of 4-5 $\mathrm{ms}^{-1}$ was maintained for all the schedules. 


\section{Evaluation}

\section{Surface checking}

Immediately after unloading the poles from the kiln, the lengths of all visible, individual surface checks $\geq 1 \mathrm{~mm}$ in width were measured using a measuring tape. Check depth and width were measured using a ruler and an improvised depth gauge. The depth gauge comprised of two $170 \mathrm{~mm}$ long and $1 \mathrm{~mm}$ thick wires fixed in a cork handle on one end, with a separation distance of $20 \mathrm{~mm}$ between them at the opposite end.

\section{MC gradient}

Directly after measuring the externally visible surface check parameters, a $25 \mathrm{~mm}$ thick disc (disc1) was cut at the TGL and three, $25 \mathrm{~mm}$ wide, radial strips were then cut from 3 positions approximately $120^{\circ}$ apart on disc 1 (Figure 1). From each strip, $12.5 \mathrm{~mm}$ radial length samples were cut as shown in Figure 1. MC gradient was measured immediately after unloading poles from the kiln. The samples were weighed and dried to constant mass in a laboratory oven at $102^{\circ} \mathrm{C}$. The $\mathrm{MC}$ of each sample was calculated according to the standard formula based on ovendry mass.

The MC gradient was then computed by subtracting the average MC of the $0-25 \mathrm{~mm}$ "long" (shell) from the MC in the centre of the pole (core) on the same radius. The $0-25 \mathrm{~mm}$ sample represents more or less the sapwood depth where MC is critical in order to achieve complete preservative penetration during impregnation. Of the three radial strips (Figure 1), only the one with the steepest gradient rather than the average of all three strips was considered in the statistical analysis. This is because microbial degradation may begin from that side of the pole which happens to be insufficiently treated (a too high MC in a particular position leads to insufficient preservative penetration), thus creating a vulnerable locality. If average radial MC gradients are considered, the one too steep gradient (out of three) may go unnoticed especially if the remaining two are low enough to generate a permissible average.

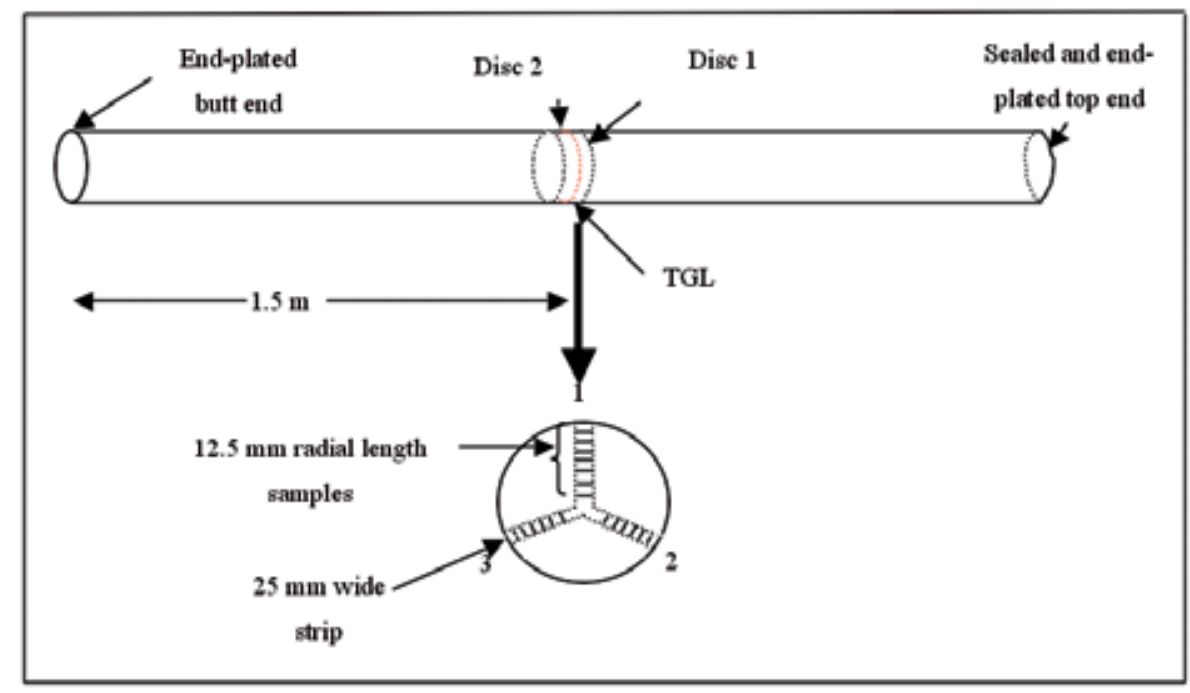

Figure 1. Pole sampling for moisture content gradient and honeycombing measurements

\section{Honeycombing}

A second disc (disc 2) was cut just adjacent to the first one (Figure 1). The exposed crosssection on the TGL side was sprayed with a solution of $0.1 \mathrm{~g}$ dimethyl yellow dye dissolved in 100 
$\mathrm{ml}$ of ethanol $(95 \% \mathrm{v} / \mathrm{v})$ to enhance the contrast of heartwood and sapwood, and scanned using a scanner (BRISA 620P VUEGO SCAN) to obtain digital images (Figure 2). Images were analyzed using Image J software (http://rsb.info.nih.gov/ij) to measure the length, maximum width and area of honeycomb checks as well as the cross-sectional area of the disc. The number of individual closed surface checks, now visible on the image of the disc cross-section, was also noted (Figure 2).

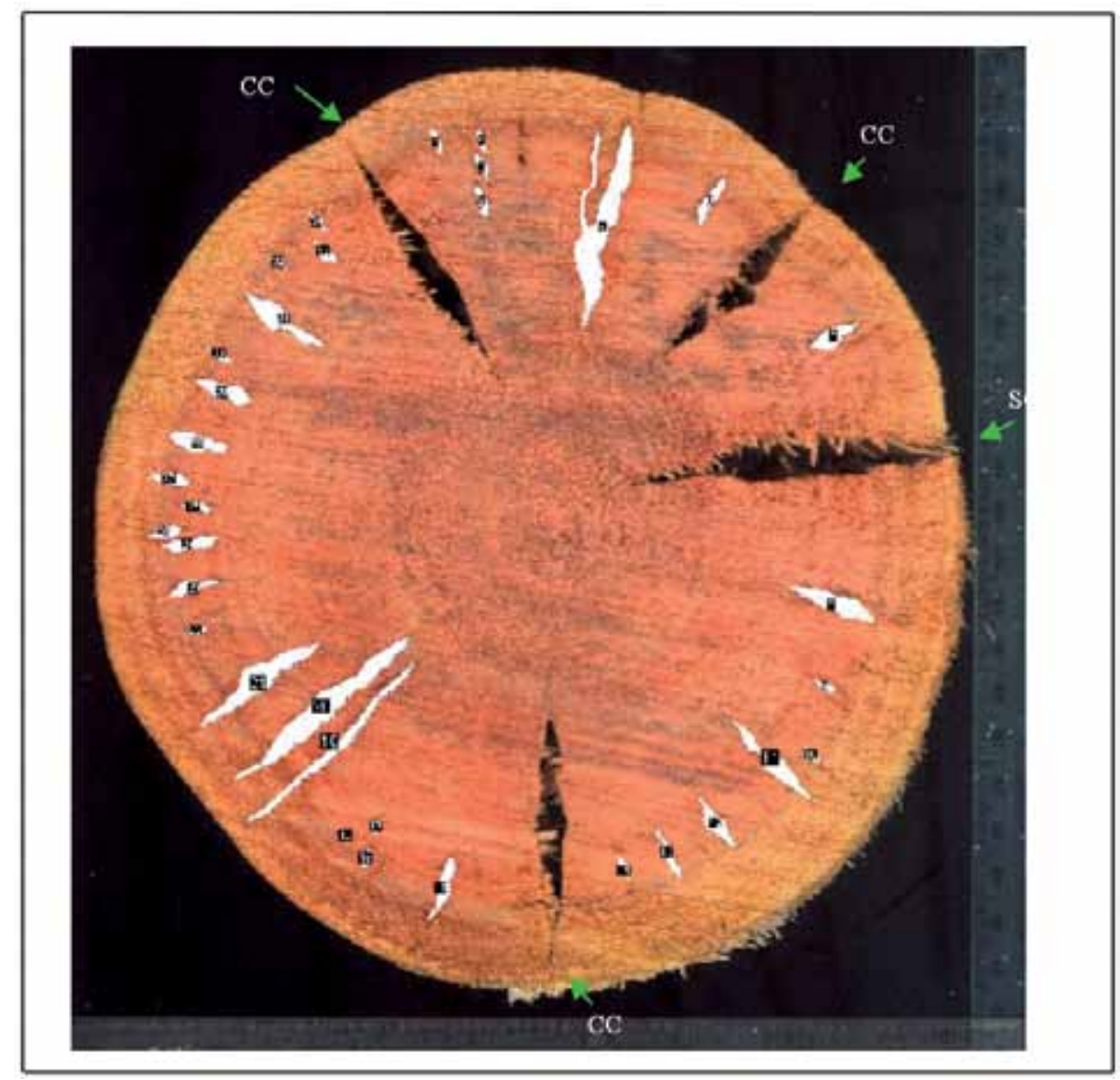

Figure 2. Cross-section image of a pole disc at TGL

Key: Filled white $=$ honeycomb

$\mathrm{SC}=$ surface checks; $\mathrm{CC}=$ closed surface checks

\section{Collapse}

Collapse was measured by assessing the outer surface of disc 2 (Figure 1) for surface profile irregularities (corrugations or grooves) developed during drying (Mugabi 2007). The disc crosssectional surfaces were also observed for presence of collapse, assessed + in terms of the degree of excessive shrinkage. The pole was then arbitrarily classified as belonging to one of the categories 1 , 2 or 3 , category 3 being one the worst and 1 the least affected by collapse.

\section{Data analysis}

A preliminary analysis of data was done to test for significant correlations among the variables related to drying defects and moisture gradients caused by the source of material and kiln schedule. Data on all thirty nine poles were then grouped together and analysed. Since the data was not normally distributed, Spearman non-parametric correlations were used. Factor analyses were also performed to isolate component factors to explain the nature of these correlations. All tests were carried out at $5 \%$ level of significance. 


\section{RESULTS}

The means and standard deviation of the MC gradients and drying defects (excluding collapse class) for both sources of poles and three drying schedules used are shown in Table 1.

Table 1: Means and Standard deviation (in brackets) of MC gradients and drying defects in poles

\begin{tabular}{|lccccccc}
\hline Source & $\begin{array}{c}\text { Kiln } \\
\text { schedule }\end{array}$ & $\begin{array}{c}\text { MC } \\
\text { gradient }\end{array}$ & $\begin{array}{c}\text { Surface check } \\
\text { count }\end{array}$ & $\begin{array}{c}\text { Surface check } \\
\text { total length }(\mathbf{m m})\end{array}$ & $\begin{array}{c}\text { Honeycomb } \\
\text { count }\end{array}$ & $\begin{array}{c}\text { Honeycomb } \\
\text { percentage }\end{array}$ & $\begin{array}{c}\text { Closed surface } \\
\text { check count }\end{array}$ \\
\hline KZN & 1 & $86.2(35.1)$ & $6.3(5.4)$ & $1.5(1.4)$ & $18.4(12.8)$ & $1.0(0.7)$ & $2.7(1.9)$ \\
& 2 & $98.4(35.9)$ & $3.1(4.0)$ & $0.7(1.0)$ & $27.0(14.4)$ & $2.6(1.7)$ & $1.7(1.7)$ \\
STB & 3 & $64.9(14.3)$ & $11.6(8.8)$ & $2.1(1.5)$ & $12.1(7.1)$ & $0.9(0.3)$ & $1.0(1.3)$ \\
& 1 & $50.3(6.7)$ & $10.1(8.1)$ & $1.5(1.2)$ & $13.9(8.1)$ & $1.2(0.6)$ & $1.1(1.5)$ \\
\hline
\end{tabular}

Key: Schedule $1=80^{\circ} \mathrm{C} \mathrm{T}_{\mathrm{db}} / 59^{\circ} \mathrm{C} \mathrm{T}_{\mathrm{wb}} / 8$ days KZN $=$ Kwa-Zulu Natal

Schedule $2=80^{\circ} \mathrm{C} \mathrm{T}_{\mathrm{db}} / 69^{\circ} \mathrm{C} \mathrm{T} \mathrm{T}_{\mathrm{wb}} / 10$ days $\quad \mathrm{STB}=$ Coetzenburg, Stellenbosch

Schedule $3=70^{\circ} \mathrm{C} \mathrm{T}_{\mathrm{db}} / 59^{\circ} \mathrm{C} \mathrm{T}_{\mathrm{wb}} / 12$ days

In the preliminary analysis, different sources of poles and drying schedules showed different defect and MC gradient correlations. A sphericity test indicated that significant correlations exist among these variables (parameters). To study these correlations for KZN poles dried with schedule 1 , a principal components factor analysis with a varimax normalized factor rotation showed that honeycomb count, honeycomb checks as a percentage of pole cross-section, closed surface checks count and collapse class were strongly correlated and grouped together (factor 1) as shown in Table 2. Surface check count and surface check total length (factor 2) were also correlated, leaving MC gradient as a separate factor 3 .

Table 2: Varimax normalized factor loadings with principal components method for KZN pole

\begin{tabular}{lccc}
\hline \multicolumn{1}{c}{ Variable } & $\begin{array}{c}\text { Component } \\
\text { Factor 1 }\end{array}$ & $\begin{array}{c}\text { Component } \\
\text { Factor 2 }\end{array}$ & $\begin{array}{c}\text { Component } \\
\text { Factor 3 }\end{array}$ \\
\hline MC gradient & 0.087063 & 0.209571 & $0.954322^{*}$ \\
Surface check count & -0.081299 & $-0.981633^{*}$ & -0.166935 \\
Surface check total length & -0.213550 & $-0.948882^{*}$ & -0.130191 \\
Honeycomb count & $0.905976^{*}$ & 0.311191 & 0.067153 \\
Honeycomb percentage & $0.862724^{*}$ & 0.295043 & 0.162719 \\
Closed surface check count & $0.805742^{*}$ & -0.096176 & -0.299079 \\
Collapse class & $0.921647^{*}$ & 0.072619 & 0.318547 \\
\% total variance contribution & 53.46 & 25.56 & 12.52 \\
\hline
\end{tabular}

Key: * = Loadings $>0.70$

Note that surface check width and depth as well as honeycomb check length and width were not included in determining correlations between defects (Table 2). Such parameters were excluded because in computing a single value per pole, averaging may be a potential source of errors. For instance a pole with just one, $4 \mathrm{~mm}$ wide surface check would seem to be in a worse condition than another pole with a $5 \mathrm{~mm}$ wide surface check and three other checks of $3 \mathrm{~mm}$ width (average $=3.5$ $\mathrm{mm}$ ). These parameters were, therefore, only used in determining the within defects correlations where dimensions of individual checks were considered.

The three factors (Table 2) represented up to $90 \%$ of the variation in the data.

The correlation structure of poles from Stellenbosch was also analysed similarly with principal component factors analysis and varimax normalized rotation. The correlations differed from that observed in KZN poles although they were dried using the same kiln schedule (schedule 1). Surface check count, surface check total length, closed surface check count and collapse class (factor 1) were correlated (Table 3). Honeycomb count and honeycomb checks expressed as a percentage of pole cross-sectional area were also correlated (factor 2) leaving $\mathrm{MC}$ gradient a separate factor (factor 3 ) as shown in a plot of factor 2 versus factor 1 (Table 3 ). 
Table 3: Varimax normalized factor loadings with principal components method for schedule 1

Stellenbosch poles

\begin{tabular}{lccc}
\hline \multicolumn{1}{c}{ Variable } & Component Factor 1 & Component Factor 2 & Component Factor 3 \\
\hline MC gradient & -0.328338 & 0.150721 & $-0.904662^{*}$ \\
Surface check count & $0.795730^{*}$ & 0.423439 & 0.334190 \\
Surface check total length & $0.824069^{*}$ & 0.074457 & 0.374067 \\
Honeycomb count & 0.023242 & $0.850092^{*}$ & -0.445013 \\
Honeycomb percentage & 0.151668 & $0.960066^{*}$ & 0.099838 \\
Closed surface check count & $0.945745^{*}$ & -0.134921 & -0.029593 \\
Collapse class & $0.761484^{*}$ & 0.322657 & 0.321118 \\
\hline
\end{tabular}

Drying defect correlations of Stellenbosch poles dried using schedule 3 differed from those observed in poles from the same source but dried using schedule 1 (Table 3). Similar factor analysis with varimax normalized rotation showed that MC gradient and surface checking (factor 1) were correlated. Correlations also existed between closed surface check count and collapse class (factor 2), and honeycomb count and honeycomb percentage of pole cross-sectional area (factor 3).

Correlation results for all thirty nine poles grouped and analysed together, irrespective of source or drying schedule are shown in Table 4. Several relationships between the variables existed, for instance surface check total length was correlated to the rest of the variables except collapse class (Table 4), hence the use of factor analysis to explain the nature of these correlations.

Table 4: Correlations of all variables

\begin{tabular}{|c|c|c|c|c|c|c|c|}
\hline Variable & MC gradient & $\begin{array}{c}\text { Surface cheek } \\
\text { count }\end{array}$ & $\begin{array}{c}\text { Surface eheck } \\
\text { total length }\end{array}$ & $\begin{array}{l}\text { Honeycomb } \\
\text { check count }\end{array}$ & $\begin{array}{l}\text { Honeycomb } \\
\text { Pereentage }\end{array}$ & $\begin{array}{c}\text { Closed surface } \\
\text { check count }\end{array}$ & $\begin{array}{c}\text { Collapse } \\
\text { class }\end{array}$ \\
\hline MC gradient & $=$ & & & & & & \\
\hline Surface check count & $-0.583^{\circ}$ & - & & & & & \\
\hline Surface check total length & $-0.489^{*}$ & $0.928^{*}$ & - & & & & \\
\hline Honeycomb check count & $0.514^{*}$ & $-0,402^{*}$ & $-0.425^{*}$ & - & & & \\
\hline Honeycomb percentage & $0.378^{*}$ & -0.310 & $-0.396^{*}$ & $0.857^{*}$ & . & & \\
\hline Closed surface cheek count & 0.013 & 0.225 & $0.322^{*}$ & $=0.004$ & -0.016 & - & \\
\hline Collapse class & 0.263 & -0.078 & -0.150 & $0.592^{*}$ & $0.733^{*}$ & $0.343^{*}$ & . \\
\hline
\end{tabular}

Key: $*=$ significant correlations $(\mathrm{p}<0.05)$

\section{Correlation between defect types and MC gradient}

A factor analysis of the data grouped together isolated three principal components factors. Collapse, honeycomb count and honeycomb checks expressed as a percentage of cross-sectional area were strongly correlated and were taken as component factor 1. MC gradient, surface check count and surface check total length were also strongly correlated (factor 2), leaving closed surface checks as a separate variable (factor 3 ) as seen in table 5.

Table 5: Varimax normalized factor loadings with principal components method for all the data grouped together

\begin{tabular}{lccc}
\hline \multicolumn{1}{c}{ Variable } & $\begin{array}{c}\text { Component } \\
\text { factor 1 }\end{array}$ & $\begin{array}{c}\text { Component } \\
\text { factor 2 }\end{array}$ & $\begin{array}{c}\text { Component } \\
\text { factor 3 }\end{array}$ \\
\hline MC gradient & 0.437 & $0.593^{*}$ & 0.085 \\
Surface check count & -0.082 & $-0.945^{*}$ & 0.111 \\
Surf check total length & -0.206 & $-0.890^{*}$ & 0.167 \\
Honeycomb count & $0.860^{*}$ & 0.334 & 0.039 \\
Honeycomb percentage & $0.920^{*}$ & 0.213 & -0.080 \\
Closed surface check count & 0.116 & -0.141 & $0.971^{*}$ \\
Collapse class & $0.864^{*}$ & 0.038 & 0.306 \\
\% total variance contribution & 49.10 & 24.48 & 10.59 \\
\hline & Key: * Loadings $>0.59$ &
\end{tabular}


These three factors represented $84 \%$ of the variation in data as shown in table 7 . MC gradient was negatively correlated with surface checking (Table 4). The plot of MC gradient and surface check total length is shown in figure 3 .

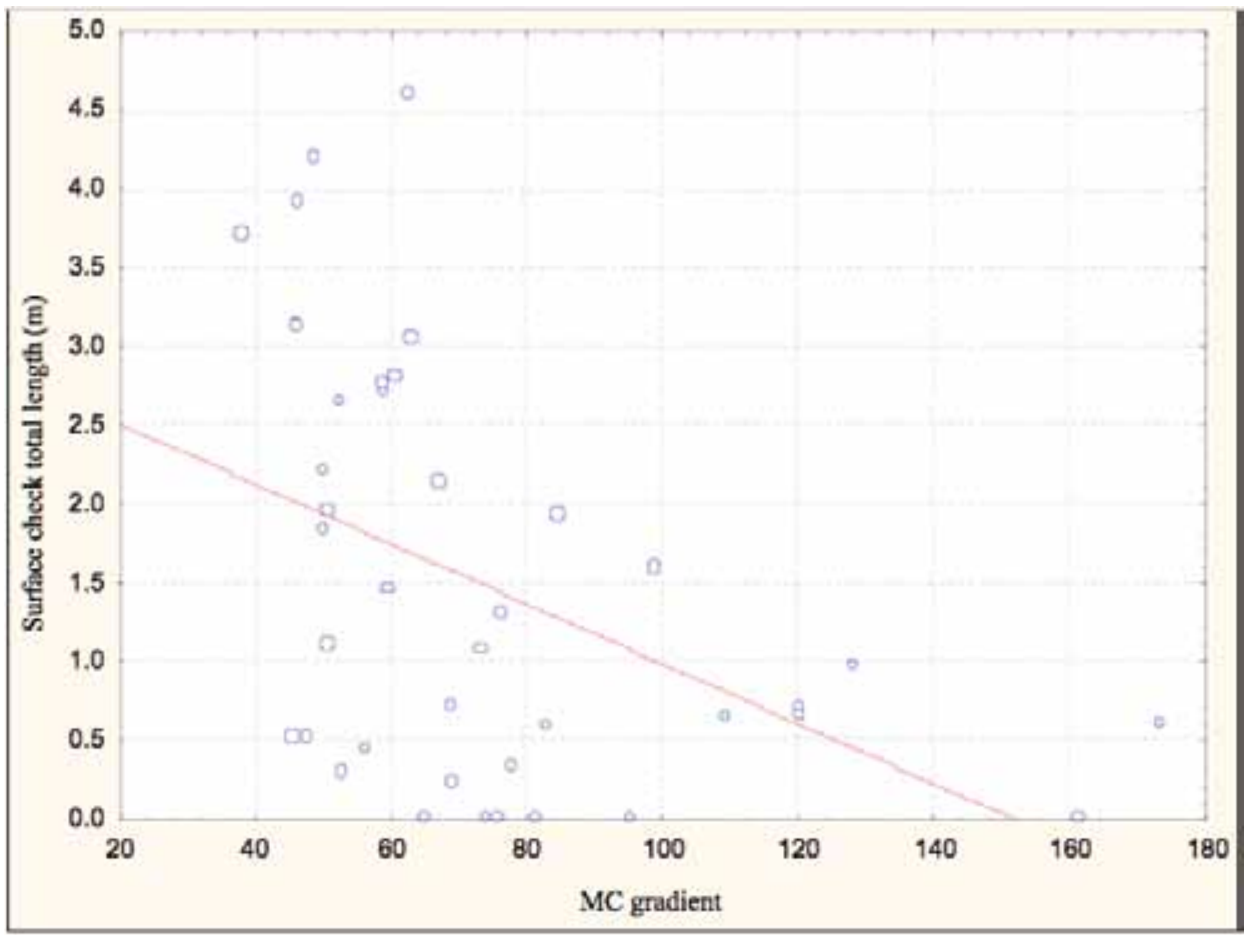

Figure 3. Plot of MC gradient and surface checking, with Spearman correlation coefficient

$$
r=-0.49(p=0.00)
$$

Where the MC gradient was very steep, MC was still high even in the outer portion, with values above fibre saturation point in the shell of some poles (Figure 4).

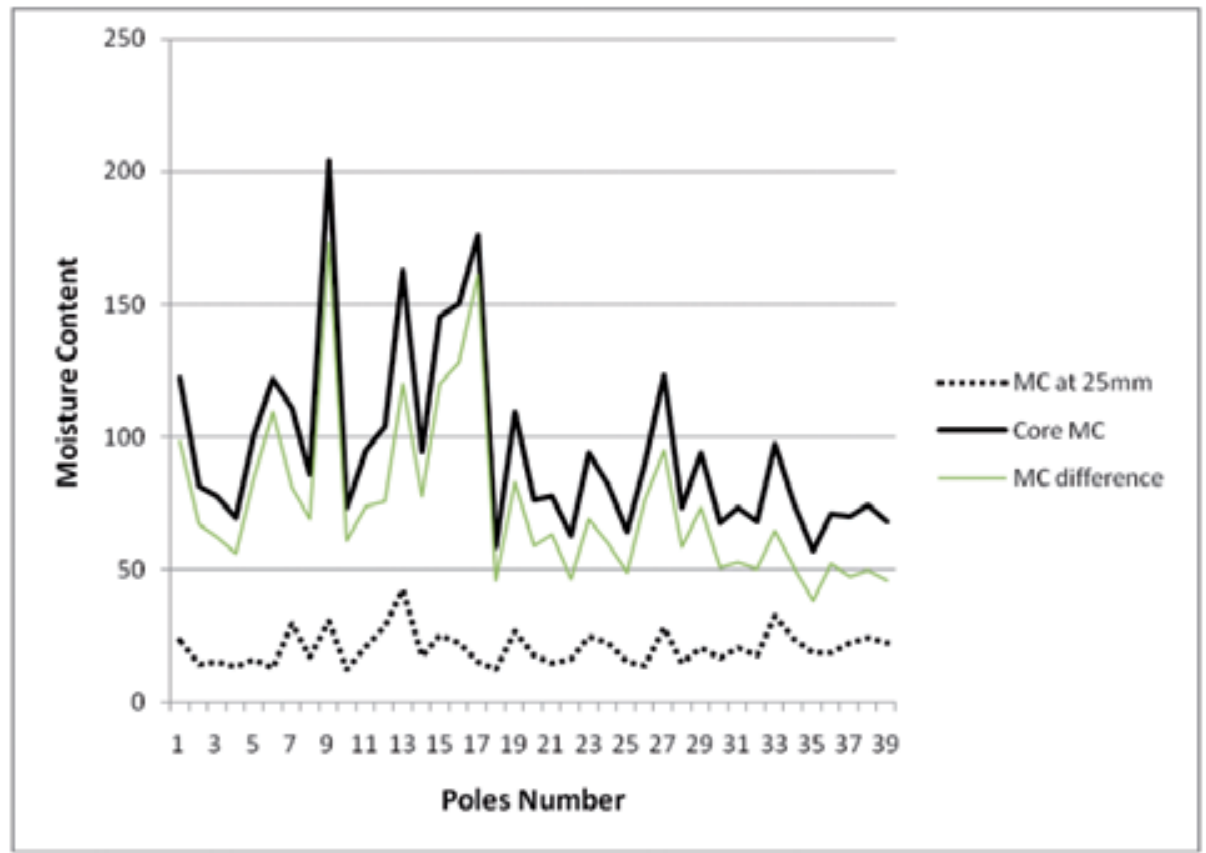

Figure 4. MC gradient between the centre and outer portion of the pole 


\section{Correlation between defect type parameters}

Correlations between length, width and depth of surface checks were not strong but significant $(0.31 \leq \mathrm{r} \leq 0.4, \mathrm{p}<0.05)$, while honeycomb check length, width and area were all strongly correlated $(\mathrm{r}$ $>0.7, \mathrm{p}<0.05)$. The correlation of honeycomb width and length is as shown in figure 5 .

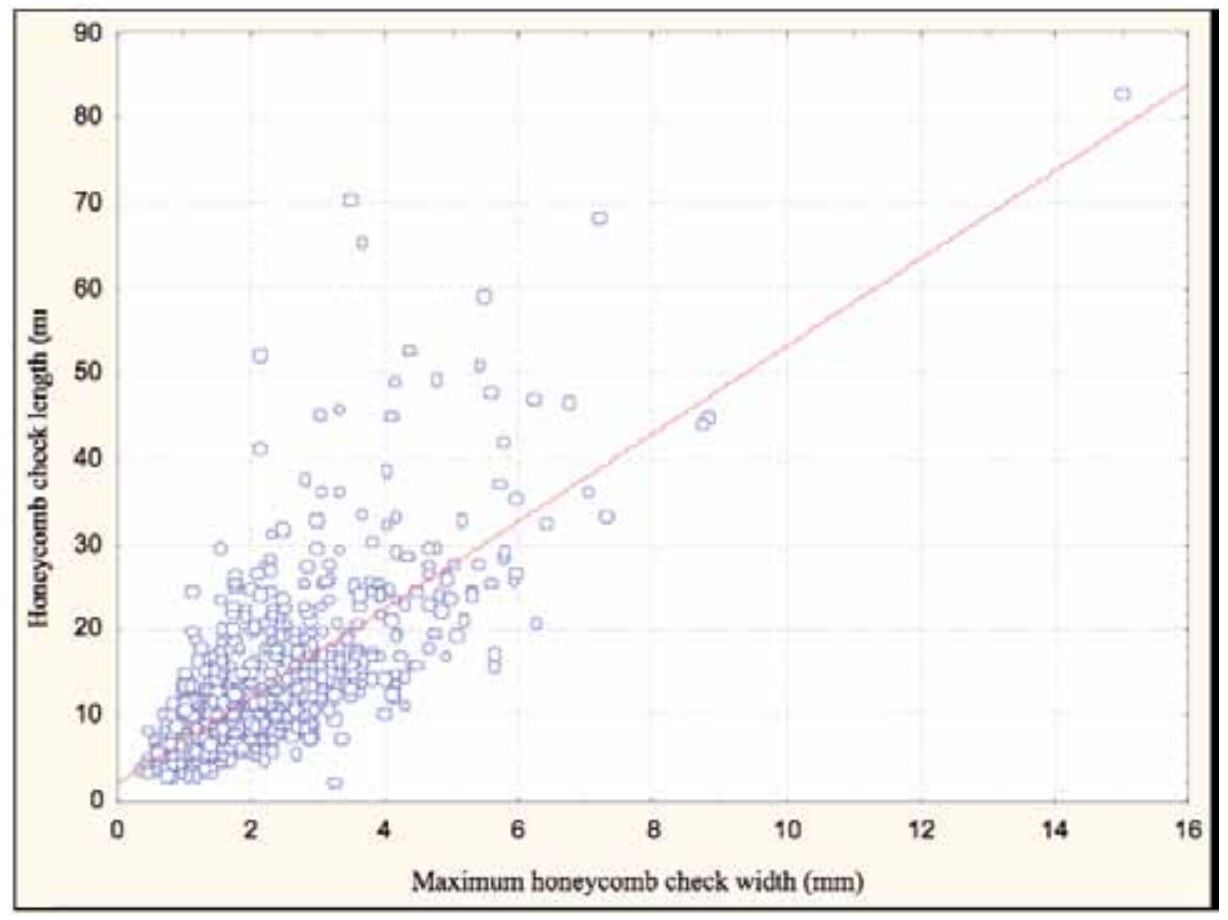

Figure 5. Plot of honeycomb check width and length, with Spearman correlation coefficient

$$
\mathrm{r}=0.70(\mathrm{p}=0.00)
$$

\section{DISCUSSION}

Variations in correlations of drying defects and MC gradients in poles from the two sources could have been caused by the different tree growth rates (and the associated growth stresses) in KZN and Stellenbosch. The combined effects of growth and drying stresses were possibly different in each case.

Varying levels of drying stresses that developed in poles dried with different kiln schedules may be responsible for the observed schedule to schedule differences in defect and MC gradient correlations. However, the limited sample used might have had an effect on the results.

Strong correlations between honeycombing and collapse corroborate observations by Brown (1989), that honeycombing may be associated with collapse where large aggregates of cells collapse and cause internal fractures. In fact, since the pole cores after kiln-drying were generally still wet with all MC's above the fibre saturation point, honeycombing most probably occurred as a result of collapse. Honeycombing associated with collapse occurs above fibre saturation point and may normally be distinguished from the internal checking occurring below fibre saturation point by the associated collapsed surfaces (Brown 1989). Chudnoff (1955) also noted that the stresses that develop during the drying of heavy Eucalyptus poles are very high and collapse is normally accompanied by severe internal checking. Based on the association of honeycombing with collapse, it seems possible to measure one of them and make reliable predictions about the other, during pole inspection. 
The correlation between surface check total length and MC gradient (after drying) between the surface layers and the centre of poles was negative. Yet Rice (1994) and Pratt (1974) noted that if MC gradient (early in drying) becomes too steep, the outer parts of the wood tend to shrink excessively on to the inner, and severe stresses develop causing surface checking in case the tensile stresses on the surface exceed the maximum tensile strength of the wood perpendicular to the grain (Hildebrand 1970). Perhaps MC gradient after drying did not relate to MC gradient early during drying and has no bearing on surface checks. It should also be noted however, that in poles where MC gradient (after drying) was too steep, the MC in the surface layers was still too high to cause appreciable shrinkage and stress development. In fact, in some poles, the MC in the outer $25 \mathrm{~mm}$ was still above the fibre saturation point and minimal shrinkage or none had occurred at all. Therefore, if treated at this moisture content, such poles would continue drying in service and new surface checks would develop and dimensions of already formed checks would also extend, exposing the inner untreated heartwood.

Although the length, width and depth of surface checks were correlated, it still is important to assess each one of them to get a comprehensive picture. For instance, surface checks may be of acceptable length and width but if the depth is not assessed and happens to be deeper than the sapwood, then the preservative impermeable heartwood is exposed. Such checks would present avenues for microbe entry into the poles in service. Although strong correlations between the length, maximum width and area of honeycomb checks were observed, measuring area as a parameter would be more reliable in cases where destructive methods are applicable.

\section{CONCLUSIONS}

Defect correlations varied with the source of poles and drying schedule used. Since a small sample was used, it is recommended that further studies be done on sufficient samples to generate reliable conclusions.

There were strong and positive correlations between honeycombing and collapse. It is possible, therefore, to make reliable deductions about one of them based on the measured results of the other.

Surface checking was found to be negatively correlated with MC gradient (after drying) although the opposite is expected with MC gradient early during drying.

Despite the positive correlations between width, length and depth of surface checks, it remains important to measure all three parameters to get the true picture of the defect condition.

Honeycomb check width, length and area were positively and strongly correlated suggesting a possible prediction of one parameter from the other. Therefore, a method that effectively measures any of the dimensions would be appropriate in assessing the overall condition.

Honeycomb count and honeycombing expressed as a percentage of pole cross sectional area were also correlated.

Surface checking was not correlated with honeycombing and collapse, thus prediction of internal defects based on surface checking is not possible.

\section{ACKNOWLEDGEMENTS}

The study was made possible by support from Bedson (PTY) Ltd, the Norwegian Agency for Development (NORAD) through Makerere University, Uganda, and the South African Wood Preservers Association (SAWPA). We also thank Messrs. A. Kunneke and W. Hendrikse for their technical contribution during data collection. 


\section{REFERENCES}

Bariska, M. 1992. Collapse phenomena in Eucalypts. Wood Science and Technology 26: 165-179.

Brown, W.H. 1989. The conversion and seasoning of wood. Linden Publishing, Fresno, California.

Chudnoff, M. 1955. The effect of zinc chloride on some shrinkage properties of Eucalyptus rostrata wood. Forest Products Journal 5 (2): 139-141.

Garratt, G.A. 1931. The mechanical properties of wood. John Wiley and Sons Inc. London.

Hildebrand, R. 1970. Kiln drying of sawn timber. Richard Schorndorfer, Plochingen. Germany.

Mugabi, P. 2007. Moisture content and drying defects in kiln-dried Eucalyptus grandis poles. $\mathrm{PhD}$ Forestry (Wood Science) dissertation, Stellenbosch University, South Africa.

Panshin, A.J., De Zeeuw, C. and Brown, H.P. 1964. Textbook of wood technology. Second Edition. McGraw-Hill Book Company. New York.

Pratt, G.H. 1974. Timber drying manual. McCorquodale Printers Ltd, London

Rice, R.W. 1994. Drying stresses: causes and solutions. In: Profitable solutions for quality drying of softwood and hardwoods. Proceedings of the conference sponsored by the Forest Products Society, Madison, 25-27 March 1994.

SANS 754 2007. Eucalyptus poles, cross-arms and spacers for power distribution and telephone systems. South African National Standard. Standards South Africa, Pretoria.

Wangaard, F.F. 1950. The mechanical properties of wood. John Wiley and Sons, Inc. New York. 